Voix et Images

voixetimages

\title{
L’appel du Nord dans Neige noire : La quête de Narcisse
}

\section{Françoise Maccabée-Iqual}

Volume 5, numéro 2, hiver 1980

Yves Thériault

URI : https://id.erudit.org/iderudit/200213ar

DOI : https://doi.org/10.7202/200213ar

Aller au sommaire du numéro

Éditeur(s)

Les Presses de l'Université du Québec

ISSN

0318-9201 (imprimé)

1705-933X (numérique)

Découvrir la revue

Citer cet article

Maccabée-Iqbal, F. (1980). L'appel du Nord dans Neige noire : La quête de Narcisse. Voix et Images, 5(2), 365-377. https://doi.org/10.7202/200213ar d'utilisation que vous pouvez consulter en ligne.

https://apropos.erudit.org/fr/usagers/politique-dutilisation/ 


\section{L'appel du Nord dans Nelge nolre: La quête de Narcisse}

Depuis des temps immémoriaux, le lointain Nord fascine l'imagination de l'homme. II y a eu l'ultima Thula des Anciens, extrême limite septentrionale du monde connu, l'Hyperborée des Grecs, sise au-delà du vent du nord, ou encore, plus récemment et plus près de nous, les pays d'en haut du coureur de bois et du voyageur canadiens. Que le Nord, au titre de contrée fabuleuse, séduise l'imaginaire québécois, le livre de Jack Warwick ' en témoigne. S'il en est ainsi, comme le souligne l'essayiste, c'est que le Nord, qui peut coincider avec une région géographique, correspond intrinsèquement à un espace mental, d'où l'importance de déceler dans une cuvre littéraire la présence de l'esprit du Nord, que cet esprit s'appuie ou non sur une toile de fond géophysique.

L'esprit du Nord participe de la quéte, elle-même mobile principal du voyage intérieur ou extérieur: "Le voyage ne se borne jamais a un simple déplacement dans l'espace: c'est davantage l'expression d'un désir ardent de découverte et de changement [...] Essentiellement, voyager c'est chercher ".2 Voilá brièvement condensé le légendaire vague à l'âme de nos "voyageurs". II en découle que l'exploration du Nord a, outre sa signification sociale en tant qu'idéal de la collectivité québécoise, une signification psychologique au niveau de l'individu. Cette composante psychologique, Warwick ne manquera pas de ta signaler à la suite d'un Arthur Buies évoquant "les lointaines et ténébreuses profondeurs" d'une région alors interdite ${ }^{3}$ : "Pour beaucoup d'écrivains canadiens-français, cette incursion utopique dans le Nord constitue la cadre idéal de leurs introspections les plus pénétrantes [...]" 4 . Qu'en est-il chez Hubert Aquin? C'est ce que nous approfondirons dans les pages à venir par une psychanalyse du texte de Noige noire, là oú le Nord, esprit et lettre, souffle le vent dans les voiles.

A proximité du cercle polaire, le Nord géographique où se déroule l'essentiel de l'intrigue du récit dernier rappelie, comme l'ancienne Thulé, la limite extrême où finit ce monde et oủ commence un autre monde, lieu inaccessible au sein d'une " contrée irréelle jailli(e) des quatre points cardinaux $m 5$. Puisque le périple en terre septentrionale répond au désir de changement du protagoniste-narrateur, à son origine se discerne la quête dans le sens tantôt 
invoqué: «Je tiens absolument a ce nous partions tel que prévu; cela marquera une coupure entre avant et apres $[\ldots]$ Notre vie va changer $[\ldots]$ même sur le plan professionnel, tout va changer. Avant, j'étais comédien: après notre voyage, je ne le serai plus [...] Fortinbras est mon dernier rôle..." (p. 16-17). Le changement, Nicolas Vanesse va le chercher au pays de Fortinbras en compagnie de sa jeune épouse, suggérant de ce fait un prolongement dans la réalité du dernier rôle dramatique. Ne faut-il pas qu'un comédien octroie au héros qu'il incarne l'envergure dont un géniteur le prive car si « le rôle de Fortinbras est pauvre, Je personnage (est) riche" (p. 11)?

La projection de Nicolas en Fortinbras est capitale d'autant qu'elle s'inscrit dès l'ouverture du récit. Grâce à elle, il s'identifie à un personnage imaginaire, idéalisé, de tradition héroïque, ce qui renvoie à un idéal du moi tout-puissant et à la prépondérance de l'imagination dans sa vie. Sous ce rapport, ce n'est pas hasard que ces repliques de Hamlet se glissent dans son scénario : “ Je suis semblable à l'ombre [...] Le monde est un rẻve et le rève est un monde..." (p. 175). Dès lors, comment s'étonner qu'une brume onirique enveloppe un paysage nordique oủ l'on cótoie tantót des glaces qui nagent "comme des poissons mystiques" (p. 88 ), tantôt "une vallée magique et bienheureuse, une combe blanche ensemencée de pyramides" (p. 89). Au reste, la seule intention de Nicolas de rédiger un scénario de film manifeste d'emblée cette emprise de l'imaginaire car, par ce biais, le scripteur travesti en metteur en scène peut a loisir métamorphoser son autobiographie en "regards et jeux dans l'espace". Conçue sur le modéle d'“ une arabesque infinie" (p. 180), " la fiction (y) est inextricablement mêlèe aux mailles de la réalité * (p. 243) et ses motifs et images s'entrelacent et se dérobent comme dans les rêveries. Tel quel, récit-incantation, le film autobiographique ne mise pas sur la fonction de représentation mais vise "l'expressivité formelle* (p. 233) et à aiguiller l'imagination du spectateur/lecteur:

Les gens devraient aller au cinéma masqués [...] Le masque, visage de pure convenance, aurait pour effet de libérer totalement le spectateur, le déchargeant des contraintes de son identité, lui épargnant de cacher sa joie ou de feindre son émotion [...] L'idéal, même, serait d'imaginer le lecteur d'un livre portant un masque (p. 159. Voir aussi p. 233).

Face á cette référence au déguisement, comment nous échapperait-il désormais ce masque du roman costumé en scénario de film ensorceleur? Dans ce cas, que camoufle la fascination de l'imagination chez Nicolas, fascination à la source autant des voltiges du scénariste que du choix par l'époux d'une "île irréelle" (p. 102) comme site du voyage de noces? En quête d'élucidation, faisons nôtre l'interrogation de Nicolas/Fortinbras : “Où donc est ce spectacle? ". A la lueur des paroles ci-haut citees, il vient à l'esprit que les profondeurs abyssales de Nicolas sont le lieu du spectacle, ce qui est dire que celui-ci se compose d'ombres constituant "l'envers d'un decor": "The play within the play". comme dans Hamlet" (p. 147). Dans la mise a jour du réseau clandestin qui alimente une fiction. le commentaire dénonçant la facticité du décor mis en scène retient l'attention puisqu'il est invitation à 
parcourir, par delà le paysage extérieur d'une odyssée en terre vierge et fabuleuse, le paysage intérieur qui se voile au regard à l'instar de Pyramiden:

Le spectateur est amené à ne pas plus croire qu'Éva à l'existence d'une enclave italienne donnant sur la mer de Barents et, ce qui frise l'automutilation, à douter de l'existence de cet archipel du Svalbard que le cinéaste a inventé, de toutes pièces, pour s'y faire dérouler l'action [...] L'enclave italienne conduit à la tacticité du Spitzbergen qui mène, par induction, aux massifs crevassés de l'Alaska, plantés dans la mer de Barents par un cartographe délirant [...] Inhabité. le décor devient insidieusement taxé d'irréalité et, par un effet en miroir du cauchemar de Nicolas, ce décor devient transparent à tel point que son identité usurpée se laisse deviner, el qu'il n'est plus, pour les spectateurs prévenus, que l'envers d'un décor (p. 145-146).

Les mots et expressions soulignés esquissent déjà l'envers du décor. Afin de mettre en relief ces contours estompés, nous allons maintenant situer la dénonciation en fonction du cauchemar avec lequel elle se présente en continuité.

En cours de cauchemar, Nicolas émet des plaintes et des cris comme si on le torturait, ce qui laisse croire que son inconscient emprisonne angoisse et souffrance. Qu'en est-il du contenu de ce mauvais rêve? II y est question de la représentation non terminée de Hamlet dans un théảtre illuminé, lequel est sis dans une enclave italienne sur les bords de la mer de Barents. Cette enclave a de particulier son isolement du reste du monde et une architecture concentrique: "Les avenues partent du centre comme des rayons, les rues sont circulaires et recoupent les avenues. La place centrale est un vrai chefd'ceuvre... (p. 144). Au registre psychanalytique, il emane d'un relevé des constituantes du rêve de Nicolas une glorification du soleil, symbole du père, et une hantise du sein maternel. Le soleil magnifié, ce sont la référence à I'Italie, le théâtre illuminé, le plan de la ville, lo site en Laponie, région ici indissociable des jours sans nuit de l'été polaire. L'obsession maternelle, ce sont l'enclave, la place centrale, la mer de Barents, la neige et Hamlet, fils incestueux de Gertrude ${ }^{6}$.

Il frappe à l'examen que la célébration du soleil traverse et pénètre le récit entier de Neige noire. Elle s'infiltre dès son commencement lors de la description de la chaleur caniculaire (p. 7), perce ensuite dans l'association de Nicolas à Fortinbras, fils vengeur du père, ou dans la croisière au Svalbard et persiste jusqu'à la fin qui évoque la communion au Christ, lumière cèleste. Dans ces exemples, il s'agit du soleil à son apogée, d'où son association au père suscite l'image d'un être fort qui éclaire, féconde, rayonne et guide. Or, il est révélateur que, des deux pères que le récit met en scène, l'un, père réel, Michel Lewandowski, l'autre, père spirituel, le narrateur-auteur Nicolas Vanesse, seul le second corresponde au pére glorieux: "Le spectateur se trouve, ni plus ni moins, dans la position tcetale, porté par le film qu'il regarde ou par les personnages; ce qui arrive à l'être porteur se rend jusqu'à lui, ce qui nourrit son géniteur le grise, ce qui l'éclaire l'éblouit» (p. 64). 
Mentionnons au passage, car cela recoupe le propos sur le prestige de l'imagination, l'exaltation du pére fictif au détriment du pere réel. Si le premier se manifeste par l'omniprésence de ses commentaires, le dernier brille par son absence tant dans l'ensemble du récit qu'auprès de ses filles Charlotte et Sylvie en temps opportun (p. 153-154). Aussi, fort d'une paternité idéale engendrée par l'écriture. Nicolas repousse la paternité biologique: "Sylvie: Si j'ètais enceinte de retour a Montréal... - Nicolas : Tu n'as que 22 ans mon amour, ça peut attendre encore... $n$ (p. 62). Cette dualité est intéressante en ce qu'elle découvre une fuite du père parallèle a la convoitise de ses attributs. II n'est pour s'en convaincre que d'invoquer les situations de rivalité masculine qui, à l'occasion, ponctuent le récit. II y a celle oú Nicolas ridiculise la vedette Jean-Louis Roux en l'imaginant dans le personnage du pére spectre plutót que dans celui de Hamlet (p. 10), celle où il règle ses comptes avec Stan Parisé en abusant de Linda (p. 11-13), celle enfin où il sacrifie Sylvie sans affronter Michel Lewandowski. Que recéle cette fuite sinon l'évitement du combat cedipien? En effet, au lieu de s'engager dans une authentique compétition avec un rival œdipien. Nicolas tente d'en triompher sur un mode que Grunberger qualifie de narcissique magique ${ }^{7}$. Conformément au subterfuge, il met inconsciemment en ceuvre une stratégie dans laquelle la lemme est instrumentale, ce qui nous amène à considérer le rôle de celle-ci dans sa vie.

La femme s'incarne dans Neige noire sous les traits de la trinité Sylvie/Eva/Linda, trinité qui évolue dans les pas d'Ophélie, personnage imaginaire vers lequel elle converge. Il existe, en ce qui a trait à la première de ces femmes, Sylvie, une filiation entre elle et le soleil que désigne son énigmatique pendentif-ostensoir, filiation qui signifie pour Nicolas une usurpation d'identité comme l'atteste sa virilité blessée à la suite des coups de pendentif que Sylvie lui inflige au pénis". Tuer la femme vise en pareille conjecture a recouvrer son bien et a inverser la situation humiliante d'un époux dont l'épouse pratique l'inceste. Par le meurtre, donc grâce à la personne interposée de Sylvie, Nicolas, d'une part, élimine magiquement te père Michel Lewandowski - lequel finit par se suicider - d'autre part. rétablit son identité virile en châtrant à son tour celle qui l'a châtré puisque " cette castration de l'autre a la valeur de l'acquisition d'un pénis personnel dans l'inconscient " ( $N$. p. 245): *Gros plan: de sa main gauche, il (i.e. Nicolas) écarte les grandes lèvres. On distingue le brillant d'une lame qui entre dans le vagin et touche te clitoris. Il procede a une introcision (p. 237) ${ }^{9}$. Le seul projet du meurtre transforme Nicolas en Père justicier, aussi, dés l'arrivée en Norvège ou le projet verra jour, sa proximité avec Dieu se transmet par l'euphorie qui l'envahit et par l'abolition du temps sur laquelle débouche l'escale de Trondheim (p. 59, p. 60).

D'où vient la méprise que la promesse implicite de divinité s'accomplisse au détriment de Nicolas, rempli de noirceur, et à l'avantage de sa victime qui se métamorphose en lumière: "Sylvie Vanesse, tu as été aveuglée par la lumière... Et maintenant, tu nous as quittés pour un royaume où tout est diaphane. Rien n'est opaque lá où tu te trouves, et la lumière rencontre la 
lumiere..." (p. 154)? Tout se passe comme si Nicolas en sacrifiant Sylvie s'amputail de son propre corps car la compagne se confond avec le miroir dont la fonction est. à l'exemple des glaces dans lesquelles Nicolas se regarde si souvent, de refléter l'image du compagnon. C'est le stade du miroir comme le suggère le rapprochement d'une description de Nicolas avec les paroles de Lacan qui, analysant la réaction du petit homme face à son image. note, entre autres observations, le plaisir ludique retiré des gestes faits devant le miroir:

II se regarde dans le miroir en faisant toutes sortes de mimiques: il prend des attitudes, se donne des airs et ne cesse de s'observer tout ce lemps. Un vrai comedien! ( $\rho$. 14-15).

Le petit d'homme à un âge où il est [...] dépassé en intelligence instrumentale par le chimpanzé, reconnait pourtant déjà son image dans le miroir comme telle. Reconnaissance signalée par la mimique illuminative [...] Cet acte [...] rebondit aussitót chez l'enfant en une série de gestes où il éprouve ludiquement la relation des mouvements assumés de l'image à son environnement reflété $[. . .]^{10}$

A maintes reprises, la jeune femme blessée par la vie éveille lidée d'Écho répercutant la voix de Nicolas/Narcisse ou celle de la fontaine dans laquelle il se contemple, aussi Sylvie lui soulignera-t-elle qu'il n'y a pas coïncidence entre ce qu'elle est et ce qu'il avait imaginé à son sujel : " Je ne ressemble pas a l'image que tu t'étais faite de moi [...] tu voudrais que je ressemble a la femme de la vie. Vu sous cet angle, l'amour a quelque chose de sinistre et de désespérant o (p. 73). Sylvie réfléchit une radieuse sensualité, d'où Nicolas obtient la satisfaction narcissique sous forme d'amour comme il l'obtient par ailleurs sous forme de film autobiographique. La femme élue constitue en somme une projection extérieure de sa féminité inconsciente non intégrée, projection qui ne saurait ternir sans risque l'image qu'il lui revient de refléler. Quand celte femme déclenchera une blessure narcissique, la faute s'avoue impardonnable (p. 131) et le gâchis irrémédiable (p. 236). La blessure provoquera, à titre de défense, le sadisme de l'offensé. Cette réaction a l'offense, évidente dans la situation d'infidélité de Sylvie, se dissimule encore dans le traitement que Nicolas réserve à Linda puisque la comédienne n'est en ce cas qu'un prolongement de Stan Parisé. Le commentateur résume cet investissement narcissique de l'autre lorsqu'il inscrit la fonction de masque et de miroir que la femme revêt aux yeux de Nicolas, Ionction analogue selon lui à la perception du réel dans un film, par définition projection d'images successives s'adressant au regard:

Linda est un personnage-écran: elle reflète. Mais rien ne passe à travers elle, surtout pas la lumière. Nicolas la regarde sans arrét [...] II est confiné à sa surface, à son air distant, à son masque. Le film coïncide très bien avec cette perception corticale du réel. Cette spécificité cinématographique $[\ldots]$ ressemble à l'aventure aléatoire de chaque individu [...] L'ètre humain, si doué soit-it, reste toujours à l'extérieur de ce qu'il veut percer, tout comme Nicolas [... (p. 23).

Le rôle de miroir et le sort de sacrifiée réservés aux femmes incitent à rechercher f'image sous-jacente de la Femme, ce qui convie à dévoiler la 
"figure cachée" de la mère dont la présence s'immisçait dans le cauchemar de Nicolas ci-dessus rappelé. Bien que le récit exclut la mère concrète (Charlotte est une future mére el Mme Lewandowski vit en retrait du monde), il se réfère à l'archétype de la mère de sorte que l'on peut écrire a son sujet ce qui est écrit de l'un de ses symboles, la terre natale: a dans le film, on peut dire que le Québec est en creux. Son éclipse récurrente fait penser à l'absence d'une présence, à un mystère inachevé..." (p. 136). Parmi les nombreux traits caracteristiques d'une fixation maternelle, 11 l'un d'eux, la croisière dans la mer de Barents, est remarquable en ce qu'il se rallie au symbole de la Traversée tel qu'on le retrouve dans les légendes anciennes et modernes:

Un héros s'engloutit dans l'eau. Il part de l'Ouest (coucher du soleil), vers l'Est, (lever du soleil = renaissance et nouvelle vie). II accomplit sa traversée dans le ventre d'un poisson (comme le fit Jonas), ou dans un bateau, ou dans une arche (comme Noe), etc. Le theme est toujours le même: le héros traverse l'eau (qui symbolise l'Inconscient), dens lo ventre d'un monstre (= le * sein maternel ". l'enfance, le passé). Le héros part vers la lumiere montante (il renait à une nouvelle vie); il sort du ventre du monstre (il sort du " sein maternel ", devient adulte). Souvent, il allume alors du fou (= conscience adulte, spiritualité). ${ }^{12}$

Que le voyage au Svalbard erre à proximité d'une frontière à franchir, l'étiquette de "voyage de noces "qui lui est accolée le confirme du fait que celui-ci désigne le rite de passage entre l'enfance et l'âge adulte. La traversée anticipée ne se réalisera cependant.pas comme l'infére cet extrait de commentaire:

Son voyage (i.e. celui du couple) est lent, et plus qu'un voyage, c'est une exploration exaltée vers le Pôle Nord, non pas pour s'y rendre, mais pour s'arrêter juste avant, à proximité de la grande banquise qui flotte comme une barrière infranchissable autour de l'absolu. Pour aider les images à charrier cette masse de symboles et de tensions, il faut ajouter à l'approche du Spitzbergen, des gros plans de Sylvie et de Nicolas comme s'ils se tenaient devant l'autel, dans une église sans pilastres, sans verrières, sans double transept, sans colonnes [...] mais non sans nef $[\ldots]$ Très peu et rien d'autre suffit pour conférer aux images du voyage dans la mer de Barents une extension planétaire $[\ldots]$ (p. 81).

Pour éclairer peu à peu cette impasse, rassemblons d'abord certains éléments contenus dans cet extrait et dans l'ensemble du commentaire: désir d'absolu en amour, cérémonie nuptiale, prolongement dans l'infini, transcendance du temps et de l'espace (extension planétaire) et fusion avec la mer/mère par le biais, d'une part, de l'immersion du couple dans une mer communiquant "avec toutes les mers, avec tous les fleuves, avec tous les affluents des fleuves et avec toutes les mers intérieures» (p. 81), par la présence, d'autre part. de la nef et de l'allusion à notre mère l'Église. Cette constellation d'éléments, jointe à l'investissement narcissique auquel la femme est sujette, implique non la sortie du sein maternel inhérente à la Traversée mais le retour au sein maternel. A cel égard, la psychanalyse 
informe que le vècu "dont l'homme recherche la répétition, c'est bien son séjour prénatal, situation dont il fut chassé sur un mode traumatisant et qu'il ne cesse pas de désirer retrouver" ( $N$, p. 24-25). Tandis qu'une vague nostalgie du sein maternel persiste chez tout être humain, elle devient hantise chez le narcissique parce que sa fixation affective plonge ses racines dans la vie prénatale selon Grunberger.

Le narcissique aspirera donc, sa vie durant, au paradis perdu, état de bien-ètre ineffable, de béatitude et de satisfaction spontanée propre au foetus. Comme celui-ci ne fail dans cel état qu'un avec la mére et le monde ambiant, il baigne dans une sensation d'existence élargie jusqu'à l'infini qui lui procure "à la fois une impression d'autonomie et de grandeur absolue" $(N$, p. 134) : voilà qui recoupe l'extension planétaire dont il fut auparavant question ! 13 En outre, comment ne pas déceler la trace de ce bonheur primitif perdu dans certains signes distinctifs des doubles de Nicolas? Sylvie porteuse de "muscles du chagrin exceptionnels" trahissant un "mal de vivre" (p. 135) qui la conduira à attenter à ses jours dans la baignoire d'une chambre d'hôtel (p. 226). Eva porteuse de loaengsel, "celte aspiration profonde de l'âme, mais à quoi... (p. 133), qui déclare qu'elle préfère le suicide à une vie sans amour (p. 199). Hamlet devenu " semblable à l'ombre ". mélancolie qui se définit, sous la plume du commentateur, * le mal d'être sur l'écorce impénétrable du réel» (p. 187).

II apparait fort révélateur que l'angoisse que suscite l'expulsion des douces eaux maternelles s'exprime a travers un cauchemar de Nicolas aux constituantes des plus symboliques. Le cauchemar se situe au moment ou Nicolas arrive à Montréal après la croisière sur le Nordnorge " dans le ventre du Spitzbergen n (p. 88). En réalité, il est en auto-taxi, en rêve, il survole le Magdalenefjorden en hélicoptère:

Rendre la caméra vomitive. Véritable cauchemar, la course de Nicolas en taxi se déroule en hélicoptère et la chaleur humide de Montréal semble émaner de l'in/andsis du Spitzbergen [...] L'image se resserre sur un gros plan de Nicolas toujours endormi. Un bruit de percussion violente et une secousse de l'auto le tirent de son sommeil : il ouvre les yeux et se couvre aussitót la figure pour se protéger [...] Une explosion se produit tout près. Les carrosseries se fracassent. Nicolas est projeté sur le cóté opposé de la banquette. On entend des cris de douleur ou de panique (p. 150-151). ${ }^{14}$

De ce choc, Nicolas sortira blessé. II hérite d'une fracture de la main gauche et ce, au moment venu de la rédaction du scénario comme si la blessure subie devait laisser une empreinte sur son travail créateur. De plus, parce que la narration signale l'inconvénient de la fracture lors du tête-à-tête de Nicolas avec une figure maternelle, à savoir Charlotte, "enceinte de plusieurs mois" (p. 152), et Eva en qui fusionnent la première femme et la Vierge Marie (p. 167), un lien se profile entre la chute de Sylvie dans le précipice que Nicolas provoque et la chute de l'enfant dans un monde où il a la sensation d'être rejeté : “C'est tellement triste l'enfance..." (p. 62). 
L'insertion d'extraits de Hamlet et de considerations historiques ou personnelles sur la piece donnent toule son ampleur a celte sensation occultée du rejet de la mère. Dans le cadre du a dévoilement graduel de l'histoire de Fortinbras " (p. 157), le jalon initial pose l'envers du visage de conquérant d'abord gravé : “ A compter de maintenant, ne plus taire la vérité sur Fortinbras qui, depuis le début, fait figure de héros rejeté * (p. 157). Cette amère constatation s'inscrit dans une réflexion de Nicolas sur l'obsédant cauchemar qui ne cesse de le harceler: sa blessure au pénis. Venant de revivre en réve l'épisode du pendentif dont Sylvie se sert pour le castrer, "Nicolas, manifestement somnambulique [...] fait une chute de tout son long 2 (p. 156). Ces scènes imputent sans conteste à la femme castrante la responsabilité de la vacillation, ce qui laisse supposer que l'image de la mére, source originelle de frustration, se perpétue dans les autres femmes. Ceci émerge des subséquents passages relatifs à la tragédie shakespearienne.

Le premier d'entre eux montre Hamlet en proie au plus sombre désespoir et réclamant vengeance. II tient a Ophélie des propos hargneux semés d'injustes reproches (p. 167-170). Ces blâmes non fondés à l'endroit de celle qui l'aime ne se comprennent qu'á la lumière de la filiation qu'opère Hamlet dans son esprit entre Ophélie el Gertrude, la mère traîtresse, infidéle et coupable d'inceste qui préfère le frère usurpateur Claudius au mari détenteur des attributs du héros solaire: "Ce sont les portraits de deux frères! Deux êtres si dissemblables... ( $(\rho .171)^{15}$. II revient au lecteur de Shakespeare de combler les points de suspension qui émaillent ces bribes car alors se superpose à l'image de Gertrude celle de Sylvie, épouse dèvoreuse que l'époux transforme, à l'heure de la mort, en mère nourricière par l'acte de lui teter les seins et par celui de consommer sa chair et de boire son sang. De manière générale, il y a répercussion du passé perturbateur dans la chronologie du récit, lequel progresse au milieu d'anachronies évoquant un temps révolu empreint d'ombres menaçantes.

L'une d'elles à peine effleurée. le motif du trère rejeté, refait surface au prochain épisode concernant Hamlet, lequel se compose principalement d'un long commentaire qui se déclare la trame invisible enchâssant le scénario que le lecteur lit depuis le début (p. 193-196). Il y est contié que Fortinbras, de son vrai nom Amlethe, était fils maudit voué à la disparition parce que jumeau de Hamlet, nommé Amlethus dans la version ancienne ${ }^{16}$. Adoplé en secret par le roi Fortinbras, ce souverain lui donna son nom, l'institua son héritier dynastique et le persuada "qu'a la mort de Claudius ou de son jumeau, il n'avait qu'à se démasquer pour devenir roi du Danemark» (p. 193). C'est ce que fit Amlethe/Fortinbras, mais son règne u fut de courte durée et par une ambiguitè de l'histoire il fut enterré sous le nom de Fortinbras dans son propre pays et à Undensacre, l'endroit même où se rendait le petit Amlethe quand il a trouvé la mort au large des côtes danoises» (p. 194).

Les sous-entendus abondent dans ces considérations sur le sort de Fortinbras: rejet du fils par la mère, alliance de la victime avec un père bienveillant et héroique, mission de conquérant du fils, retour au pays natal qui voile un retour au sein maternel comme en témoigne l'évocation des 
"relations secrètes que la reine Gertrude aurait eues avec Fortinbras à Undensacre” (p. 195). C'est le rejet d'Amlethe qui éclaire l'absence d'intégration du narcissisme chez Nicolas Vanesse, “Fortinbras au second degré " (p. 195), car il avertit d'une défaillance de la mère, principale responsable de cette intégration. C'est en effet sur les parents, en particulier sur celle avec qui il est censé vivre en réciprocité, que l'enfant s'appuie pour accepter et conquérir l'univers où il est éjecté. Dans cet apprentissage, l'amour de la mère constitue l'apport narcissique grâce auquel s'établit la continuité entre la vie pré-natale et post-natale, apport décisil dans l'adaptation progressive au principe de réalité. L'amour empéché, insulfisant ou différé, privant l'enfant d'une confirmation essentielle, représente une frustration qui renforce la blessure narcissique primitive puisqu'il met en échec la toute-puissance originelle. Par la suite, l'être doublement blessé non seulement ne pourra plus solliciter les gratifications narcissiques de manière adaptée et efficace, mais il demeurera en mal de réintégrer le monde fusionnel du narcissisme prénatal, de caractère illimité, intemporel et tout-puissant.

Dans ce cadre, la valorisation du phallus et du Père discernée chez Nicolas, à lorigine de sa projection en Fortinbras et de son voeu d'être géniteur du lecteur, découle d'un besoin de confirmation narcissique de l'étre lésé. Ce dernier l'obtient en passant par l'intermédiaire d'une figure paternelle idéale qui compense en quelque sorte son sentiment d'impuissance fondamentale. A ce sujet, Grunberger spécifie que l'inconscient vit l'intégrité narcissique comme un coit et la blessure narcissique ou la dévalorisation comme une impuissance sexuelle, aussi l'idéogramme par lequel le langage de l'inconscient traduit celle-ci est-il le phallus sous sa forme négative, celui manquant ou endommagé, c'est-à-dire la castration:

Le phallus est un pont réalisant la complétude narcissique, comme il réunit les deux membres d'un couple dans le coït. II représente la virtualité de cette union ainsi que celle de la réalisation de l'intégrilé narcissique dont il est l'emb/eme ef l'image (N. p. 216)."1

A la lueur de cette constatation, combien éloquente l'ouverture de Neige noire qui conjugue chez Nicolas dépression, frustration sexuelle et blessure au pénis (p. 8, p. 9)!

Dans la relation de Nicolas avec Eva, la quête du miroir-confirmation narcissique s'incorpore plus ouvertement à l'union avec la mère que dans la relation avec Sylvie. D'une part, la liaison participe de l'inceste en ce sens qu'Eva s'offre comme sceur ou double de Sylvie et que dominent dans son intimite avec Nicolas des traits maternels tels la tendresse affectueuse et le partage bienveillant. D'autre part, leur vie amoureuse, quoique vécue intensément, est en réalité vécue sur un plan affectif plutôt que sexuel malgré sa connotation érotique. Divers indices en avertissent. Ainsi, dès sa première apparition, Eva mentionne sa solitude el l'échec de șa vie amoureuse (p. 54), lot de Nicolas après la mort de Sylvie, aussi leur réunion conjugue-t-elle deux solitudes et se mettra-t-elle au départ sous l'égide fraternelle: "Considérezmoi comme un frère, d'accord? Et je vous traite comme si vous étiez ma 
soeur... (p. 133). Bien que cette fraternité enfreigne à brève échéance les règles relatives aux interdits sexuels, elle demeure prévalente dans le róle de consultante et de collaboratrice que la Norvégienne assume auprés du créateur et du cinéaste. Méme leurs rapports physiques accusent cette prépondérance de l'affectivité comme l'indiquent l'amorce et le déroulement de la pièce érotique qui se joue au lit de concert avec la pièce tragique qui se joue à la télévision. Lorsqu'Éva commence à caresser Nicolas, elle est certes animée par un désir de consoler le compagnon en proie aux larmes et à la dépression comme l'est Hamlet (p. 168). Le ravissement qui ensuite saisit Nicolas équivaut à un retour au sein maternel, ce que fonde la transplantation en Eva, d'autant que les amants, métamorphosés a la tin en a statues de sel . (p. 176), se situent hors du temps:

Eva... je m'allonge démesurément en toi. C'est une sensation bouleversante... comme si j'étais maintenant lié à toi par une transplantalion totale et qui ne peut s'arrêter; même ma tête se gonfle de sang et mes cheveux laissent echapper des étoiles floconneuses... (p. 175).

La constante narcissique dans cette union avec Eva s'insinue, non sans éloquence, par la présence à l'écran de Nicolas, dans le personnage de Fortinbras, aux moments précis de l'ivresse.

Cet investissement narcissique des plaisirs sexuels chez Nicolas jaillit également de nombreuses scènes érotiques du fait que ces dernières relèvent fréquemment de la masturbation, à preuve les scènes d'auto, d'avion et de chambre au cours desquelles «Sylvie joue le rôle de fellatrice* (p. 43) et celles où Linda lui propose de le " faire " comme elle avait au préalable " fait " Stan Parisé (p. 25, p. 28). L'intimité avec autrui assure ainsi u l'accession à la complétude narcissique", laquelle "se confond dans l'Inconscient avec le retour au sein maternel, ce retour comportant obligatoirement le coït avec la mère, il devient donc en même temps la réalisation de l'inceste - (N. p. 50). II est à noter que cette réalisation incestueuse filtre également à travers l'union amoureuse d'Éva et de Linda en vertu de leur identification au Christ et a la Vierge et de la proximité avec Dieu, l'une supposant la réunion de la mére et du fils, l'autre présumant la substitution du fils au Pére puisque l'archiétype de Dieu est inséparable de celui du Père: "Linda: Tu es vierge et tu resteras toujours vierge [...] Le Christ s'est réincarné en toi [...] - Eva : Dieu est tout en moi, mais aussi j'entre en Dieu. J'ai le sentiment de l'habiter... (p. 251-252).

Il est par ailleurs évident que cette liaison lesbienne demeure, à l'instar des liaisons amoureuses de Nicolas, liaison narcissique, ce qui dérive de la continuelle superposition des deux jeunes femmes à la seule figure de Sylvie et se communique dans leurs voix en écho à la clôture du récit (p. 249-250). En d'anodines remarques à Éva au début de leur intimité, Linda laisse même deviner le lien de ce symbolique retour au sein maternel avec la trauma de la chute de l'enfant dans le monde: «Linda: Nous devons être bien écorchées, toi et moi $[$... J J'ai le sentiment que nous pansons des blessures..." (p. 249). II ressort de l'expansion cosmique et du caractere tout-puissant, infini, intemporel de cette communion d'amantes que la nostalgie du séjour pré- 
natal inclut, au-delà de la fusion mère-enfant, "la fusion de l'enfant avec son monde qui pour lui est / $\theta$ monde" ( $N$, p. 334) car il ne connait pas à ce stade de limite entre lui-même et son milieu ambiant:

II reconstruit ainsi son mode de vie intra-utérin où, toujours grâce à son complément qui était en même temps lui-même. il poursuit une existence autonome tout comme les amoureux dont on dit qu'ils vivent d'amour et d'eau fraiche. II forme alors un univers clos quant a ses besoins et largement ouvert quant à ses possibilités, se confondant avec le monde, ignorant l'objet en tant que tel, ainsi que sa propre qualité d'objet, son Moi et donc ses limites (N. p. 155).

En des mots poétiques, le commentateur traduira ces observations de l'analyste:

II ne fail aucun doute qu'Éva [...] a franchi le seuil de l'invraisemblance. entraînant avec elle le lecteur qui s'abandonne en esprit à ce qui la brùle dans une voûte rameuse qui ne cesse d'être indéfiniment gonflée. comme si tous ses rameaux se soulevaient synergiquement sous la poussée d'un seul et unique baiser qui n'est pas celui de la mort, mais celui de la vie et de l'amour $[\ldots]$ (p. 253).

Sous l'angle du moi ego-cosmique, la bifurcation finale de Nicolas vers l'homosexualité.travestie en tribadisme s'interprète comme tendance de l'être vers la complétude bisexuelle qui fut d'abord sienne à l'orée de sa vie. A la fois mâle et femelle, il jouissait alors d'une autonomie équivalente en somme a l'absence de différenciation sexuelle. N'est-ce pas cette dernière absence qui èmerge de l'extase d'Éva et de Linda et ce, en raison de l'identité de sexe et du mysticisme qui imprègne leur félicité? En effet. à l'exemple du narcissisme, «"l'union mystique" se réalise sur un plan préambivalent et quand sainte Thérèse se fait "transverbérer par un dard d'or" et décrit les sensations qu'elle éprouve et qui sont celles du coït, tous les mystiques comprennent que son vécu est exempt d'éléments sexuels proprement dits " ( $N$. p. 156). Si le stade du miroir est celui de l'attirance vers l'être de même sexe selon Freud, c'est peut-être aussi parce qu'il est celui où l'étre n'en est pas encore venu à accepter son unisexualité.

Au moment de boucler la boucle, il appert que l'appel du Nord dans Neige noire, voyage "en protondeur et de façon constellaire" (p. 177), conduit a l'Undensacre de Fortinbras * qui est une vision suspensive du temps» (p. 251), c'est-à-dire une soif d'intini et d'éternité qui s'enracine dans la cœenesthésie spécifique a la vie du foetus pour qui le temps n'existe pas:

Ici commence le tompus continuatus de l'ancienne Thulé [...] Le temps ne s'arrétera plus; il supprime, par le vide, tout ce qui le précède et tout ce qui le suivra (p. 63-64). 
1. L'appel du Nord dans la litterature canadienne-française, traduction de Jean Simard. Montréal, Hurtubise, collection Conștantes, vol. 30. 1972.

2. J.E. Cirtot, Dictionnaire des symboles. Cilé par Warwick, op. cit., p. 110.

3. L'Outaouais supérieur. Cité par Christian Morissonneau, “ Le Nord québécois au $X \mid X^{\circ}$ siècle: mythe et symbole ., forces, no 20, 1972, p. 10.

4. Op. cit., p. 192.

5. Hubert Aquin. Neige noire. Montréal, La Presse, collection Ecrivains des deux mondes. 1974. p. 88. Nous n'indiquerons désormais que la rétérence paginale.

6. Lire à ce sujet Ernest Jones, Hamlet and CEdipus. New York. Doubleday Anchor Book, 1954

7. Dr Bèla Grunberger, Le Narcissisme. Paris. Petite Bibliotheque Payot, 267, 1975, p. 309. Désormais désigné par $N$. Cet évitement perce dans les divers romans d'Aquin. Se rappeler, dans Prochain Ep/sode, l'incapacité du héros d'entrer en conflit avec de Heulz, dans Trou de mémoire. celle de Magnant de faire face à Ghezzo-Quénum, dans l'Antiphonaire, celle de Christine de combattre ouvertement Jean-William.

8. Nous développons cette filiation au soleil et l'usurpalion d'identité dans notre livre Hubert Aquin romancier. Québec, Les Presses de l'U. Laval, Vie des Lettres québécoises, 1978, p. 239.

9. II ressort de l'ceuvre d'Aquin que le viol el l'identité révolutionnaire anticipent ce mème résultat. Dans son Cahier noir, particulièrement dans la partıe intitulée " suite", Magnant confesse de quelle manière il supplée la puissance a l'impuissance: c'est en violant un nombre toujours croissant de belles inconnues qu'il linit par pouvoir s'identifier au révolutionnaire dont il projette l'image. Dans ces pages, Magnant ira mème jusqu'à suggérer, mine de rien, le lien d'un tel contexte avec I'homosexualité latente ou déclarée: * L'autre jour, dans une librairie, j'ai longuement hésite entre "L'homme impuissant", "La femme trigide" et "Onanisme et homosexualité" [...] * (Trou de mémoire. Montrèal, Le Cercle du livre de France, 1968, p. 116. Voir egalement p. 111-120).

10. Jacques Lacan, "Le Stade du miroir comme formateur de la fonction du Jew, Ecrits 1. Paris, Seuil, 1966, p. 89-90.

11. Voir notre relevé dans Hubert Aquin romancier, p. 245, note 11.

12. Pierre Daco, Les Triomphes de la psychanalyse. Verviers, Marabout Service 29. 1965, p. 309.

13. Dans tous les romans d'Aquin, la dimension spatiale a un coefficient de phénoménal qui entraine la disparition des frontières entre pays et continents. L'espace géographique, empreint de grandiose, de subjectivité el de tantastique, relève de l'imaginaire plutót que du réel ainsi que nous le rappelions ci-haut.

14. Dans un roman inédit de Hubert Aquin. L'Invention de la mort 1959 ? (sic), un protagoniste-narrateur obsedé par le sein maternel evoque sans travestissement l'angoisse de la naissance. Se rétérant à un cri qui l'a réveillé en cours de cauchemar, René Lallemant écrit: “ ll me semble que j'ai déjá crié aınsi comme un ètranglé, au fond d'un couloir annelé, quand $j$ 'ai aperçu une tenètre entr'ouverte sur te dehors, et que j'ai compris, trop tard, qu'il y avait une lissure dans mon univers fermè. Au fond, ce rève n'en était peut-être pas un, et se rapproche plutót d'un souvenir. J'ai revècu. l'autre nuit, l'instant, brutal et inoubliable, de ma naissance ; à travers l'écran de 29 ans d'obscuration, j'ai gémi une seconde fois au seul affleurement indistinct de ce souvenir atroce. Se n'aime pas le jour et ma venue au monde m'a traumatisé a jamais, si bien que. depuis. je ne cesse de deperir et de me sentir nu [...] Quel cauchemar que l'enfance [...]" (p. 150-151). René a d'ailleurs refusé dans le passé que sa maitresse Nathalie mette au monde leur fils, empruntant lui-mème l'argent nécessaire à l'avortement. (Cité avec la permission d'Andree Yanacopoulo.) 
15. Lire Hamlet, acte III, scène 4.

16. Hubert Aquin a dit avoir inventé cette gémellation de toutes pièces. Voilà qui persuade de l'importance du thème du frère rejete. source d'une ambivalente rivalité Iraternelle/masculine. Dans le récit premier, Les Rédempleurs, celle-ci s'articule sous son double aspect: Kénaz battra son ami Aram lorsque ce dernier lui offre son amour et se fera battre par son propre frère Héman après l'avoir lui-même attaqué. Par la suite. la rivalité Iraternelle/masculine oppose et unit le héros et de Heutz (Prochain Episode), Magnant et Ghezzo-Quénum (Trou do mémoire). Christine et Jean-William/Chigi (L'Antiphonaire), Nicolas el Sylvie/ Michel Lewandowski (Neige noire). Pour ce qui est de L'Invention de la mort. le thème du frère rejeté s'y énonce d'abord sans détour pour ensuite se déplacer vers un substitut du frère. l'ami Jean-Paul, que le protagoniste René adore el abhorre simultanément : " j'ai payé cher dans mon enfance d'ètre le second, celui qu'on delaisse. J'en porte encore la cicatrice, et je n'en linis pas de vivre en fils oublié» (p. 21). (Cité avec la permission d'Andrée Yanacopoulo.)

17. Les réflexions de ce paragraphe et du précédent s'appuient sur les analyses de cet auteur. Voir a ce sujet $N$. p. 153,209, 259, 260 et 270. 